\title{
Explicit and Implicit Complementarity Problems in a Hilbert Space
}

\author{
A. Carbone and P. P. Zabreiko
}

\begin{abstract}
We present some new results about solvability of implicit complementarity problems in a Hilbert space. We discuss two approaches. One of them is based on the usual change of variables and reduces the implicit complementarity problem to the explicit one. The second approach is based on the Skrypnik degree which, in the case of mappings in a Hilbert space, is essentially more general in comparison with the classical Leray-Schauder degree. In both cases, the solvability results are formulated in terms of auxiliary complementarity problems with parameter.

Keywords: Explicit and implicit complementarity problems, topological degree, exceptional elements, homotopy, operators of class $S_{+}$, quasi-monotone operators
\end{abstract}

AMS subject classification: 90C33, 55M25, 55M20

This article is a direct continuation to the article [1] which dealt with application of the Skrypnik degree to the solvability of explicit complementarity problems in a Hilbert space $X$. This problem consists in finding an element $u \in K$ such that

$$
u \in K, \quad-f(u) \in K^{*}, \quad-(u, f(u))=0 .
$$

Here $K$ is a closed cone in $X$ and

$$
K^{*}=\{x \in X:(x, \xi) \geq 0 \quad(\xi \in K)\}
$$

is its dual wedge (a cone if and only if $\overline{K-K}=X$ ), $f$ is an operator defined on $K$ and taking its values in $X$. In this article we consider the implicit

A. Carbone: Univ. della Calabria, Dip. di Matem., IT-87036 Arcavacata di Rende (Cosenza), Italia; carbonea@unical.it

P. P. Zabreiko: Nat. Acad. Sci., Inst. Math., ul. Surganova 11, Minsk, 220072 Belarus \& Belarus State Univ., Fac. Mech. and Math., Prospect Skoriny 4, Minsk, 220050 Belarus; zabreiko@bsu.by

ISSN 0232-2064 / \$2.50 C Heldermann Verlag Berlin 
complementarity problem; this problem consists in finding an element $t \in X$ such that

$$
g(t) \in K, \quad-f(t) \in K^{*}, \quad-(g(t), f(t))=0
$$

where $f$ and $g$ are operators acting in $X$. This problem can be considered as a generalization of (1) since in the case $g=I$ (and when $t=u$ ) problems (1) and (2) are the same. In what follows we call problem (2) the complementarity problem with operators $f$ and $g$.

The aim of this article is to show that topological methods lead to some simple statements about solvability of the implicit complementarity problem of alternative type. More exactly, in the main part of the article, assuming that the operator $g$ is of class $S_{+}$and the operator $f$ is complete continuous, it will be proved that under some natural conditions:

For some bounded domain $\Omega$, either each complementarity problem with operators $(I-\lambda) g+\lambda f \quad(0<\lambda<1)$ and $g$ has a solution on the boundary $\partial \Omega$ of the domain $\Omega$ or the original complementarity problem with the operators $f$ and $g$ has a solution in the domain $\Omega$.

In [6] (see also $[2,5]$ ) topological methods are applied to the implicit complementarity problem in the finite-dimensional case. We point out that the method suggested there is different from ours. Moreover, the result from [6] about the solvability of the implicit complementarity problem is not completely equivalent to ours even in the finite-dimensional case. We will compare the corresponding exceptional families of elements at the end of the article.

However, we continue to avoid the notion of exceptional families of elements which are essential for considerations and constructions in [6] and prefer more standard terminology of homotopy theory. As in [1] the basic technique in this article is the Skrypnik degree.

1. Recall that the operator $P_{K}$ of the best approximation onto $K$ is defined by the equation

$$
\left\|x-P_{K} x\right\|=\inf _{u \in K}\|x-u\|
$$

The basic properties of the operator $P_{K}$ are gathered in the following lemma; one can find proofs of these and other properties, e.g., in [7, 9].

Lemma 1. The operator $P_{K}$ has the following properties:

(a) $P_{K}$ is a positively homogeneous and non-expansive operator:

$$
\begin{aligned}
& P_{K}(\lambda x)=\lambda P_{K} x \quad(x \in X, 0<\lambda<\infty) \\
& \left\|P_{K} x^{\prime}-P_{K} x^{\prime \prime}\right\| \leq\left\|x^{\prime}-x^{\prime \prime}\right\| \quad\left(x^{\prime}, x^{\prime \prime} \in X\right) ;
\end{aligned}
$$


moreover, the operator $P_{K}$ satisfies the inequality

$$
\left\|P_{K} x^{\prime}-P_{K} x^{\prime \prime}\right\|^{2} \leq\left(x^{\prime}-x^{\prime \prime}, P_{K} x^{\prime}-P_{K} x^{\prime \prime}\right) \quad\left(x^{\prime}, x^{\prime \prime} \in X\right) .
$$

(b) In the infinite dimensional case $P_{K}$ is not a weakly sequential continuous operator. However, it has the following property of ws-closedness: if a sequence $\left(x_{n}\right)$ weakly converges to $x_{*}$ and the sequence $\left(P_{K} x_{n}\right)$ converges in norm to $z_{*}$, then $P_{K} x_{*}=z_{*}$.

(c) The equality $u=P_{K} x \quad(x \in X, u \in K)$ holds if and only if

$$
(x-u, v) \leq 0 \quad(v \in K) q \quad(x-u, u)=0
$$

in particular, for any $x \in X$,

$$
\left(x-P_{K} x, v\right) \leq 0 \quad(v \in K), \quad\left(x-P_{K} x, P_{K} x\right)=0 .
$$

(d) The equation $P_{K}+P_{\left(-K^{*}\right)}=I$ holds; moreover, the equality $x=u+v$ with $u \in K, v \in K^{*},(u, v)=0$ holds if and only if $u=P_{K} x$ and $v=P_{\left(-K^{*}\right)} x$.

Here $P_{\left(-K^{*}\right)}$ is the operator of the best approximation onto $\left(-K^{*}\right)$. In the most important case when $X=L_{2}(\Omega, \mathcal{A}, \mu)$ (here $\Omega$ is a set, $\mathcal{A}$ a $\sigma$-algebra of subsets, $\mu$ a $\sigma$-finite measure) and $K$ is the cone of non-negative functions from $X$, we have the evident equation $P_{K} x=x_{+}, P_{\left(-K^{*}\right)} x=-x_{-} \quad(x \in X)$ where $x_{+}=\max \{x, 0\}$ and $x_{-}=-\min \{x, 0\}$.

Now we assume that the operator $g$ satisfies the condition $g(0)=0$ and is invertible. Then the explicit complementarity problem

$$
u \in K, \quad-f \circ g^{-1}(u) \in K^{*}, \quad-\left(u, f \circ g^{-1}(u)\right)=0
$$

is similar to (1) but with the operator $f \circ g^{-1}$; observe that this problem and problem (2) with the operators $f$ and $g$ are connected to each other, namely, by the change of variable $u=g(t)$.

Thus, under the assumption about the invertibility of the operator $g$ one can reduce the investigation of the implicit complementarity problem to the investigation of the explicit one. In order to apply results from [1] we need some notations and definitions.

Let, as usual,

$$
\begin{aligned}
B_{r} & =\{x \in X:\|x\| \leq r\} \\
S_{r} & =\{x \in X:\|x\|=r\}
\end{aligned} \quad(0<r<\infty) .
$$

We will say that the pair of operators $f$ and $g$ is regular if for each sequence $\left(t_{n}\right) \quad\left(t_{n} \in X\right)$ such that $g\left(t_{n}\right) \in K(n \geq 1),\left(g\left(t_{n}\right)\right)$ weakly converges to 
$u_{*}=g\left(t_{*}\right)$ and $\left(f\left(t_{n}\right)\right)$ converges in norm to $v_{*}$, then $f\left(t_{*}\right)=v_{*}$. If a pair of operators $f$ and $g$ is not regular we introduce a multi-valued operator $\tilde{f}$ by the formula

$$
\widetilde{f}(t)=\left\{\lim _{n \rightarrow \infty} f\left(t_{n}\right):\left(t_{n}\right) \in \mathcal{Q}_{(f, g)}(t)\right\}
$$

where $\mathcal{Q}_{(f, g)}(t)$ the set of sequences $\left(t_{n}\right)$ such that $g\left(t_{n}\right) \in K,\left(g\left(t_{n}\right)\right)$ is weakly convergent to $g(t),\left(f\left(t_{n}\right)\right)$ converges in norm to an element from $K^{*}$, and $\left(f\left(t_{n}\right), g\left(t_{n}\right)\right) \leq 0 \quad(n \geq 1)$. The multi-valued operator $\tilde{f}$ will be called the special closure with respect to the pair $f, g$ of the (single-valued) operator $f$. For a single-valued operator $g$ and a multi-valued operator $\tilde{f}$ one can consider a generalized implicit complementarity problem of finding an element $t \in X$ such that

$$
g(t) \in K, \quad-\widetilde{f}(t) \cap K^{*} \neq \emptyset, \quad-(g(t), \phi)=0 \quad\left(\phi \in \widetilde{f}(t) \cap K^{*}\right) .
$$

Theorem 1. Let $f$ and $g$, with $g(0)=0$, be continuous operators in $X$, such that $g$ has a continuous inverse $g^{-1}$ and, moreover, either $f$ is compact and $g^{-1}$ is bounded or, at least, $f \circ g^{-1}$ is compact. Let $(f, g)$ be a regular pair of operators and $0<r<\infty$. Then

- either for some $\lambda \in(0,1)$ the complementarity problem with the operators $g$ and $(1-\lambda) g+\lambda f$ has a solution in the set $g^{-1}\left(S_{r} \cap K\right)$

- or the complementarity problem with the operators $g$ and $f$ has a solution in the set $g^{-1}\left(B_{r} \cap K\right)$.

Theorem 2. Let $f$ and $g$, with $g(0)=0$, be continuous operators in $X$, such that $g$ has a continuous inverse $g^{-1}$ and, moreover, either $f$ is compact and $g^{-1}$ is bounded or, at least, $f \circ g^{-1}$ is compact. Let $0<r<\infty$. Then

- either for some $\lambda \in(0,1)$ the complementarity problem with the operators $g$ and $(1-\lambda) g+\lambda f$ has a solution in the set $g^{-1}\left(S_{r} \cap K\right)$

- or the generalized complementarity problem with the operators $g$ and $\tilde{f}$ has a solution in the set $g^{-1}\left(B_{r} \cap K\right)$.

2. Now we omit the assumption about the invertibility of $g$ and begin a direct analysis of the complementarity problem with operators $f$ and $g$. Consider the family of complementarity problems

$$
\begin{gathered}
g(t) \in K, \quad(1-\lambda) g(t)+\lambda f(t) \in K^{*}, \quad(g(t),(1-\lambda) g(t)+\lambda f(t))=0 \\
(0 \leq \lambda \leq 1) .
\end{gathered}
$$

For $\lambda=1$ this is the original complementarity problem (2); for $\lambda=0$ this problem is degenerated:

$$
g(t) \in K, \quad g(t) \in K^{*},(g(t), g(t))=0
$$


and equivalent to solving the equation

$$
g(t)=0
$$

Family (3) is a linear deformation connecting the original complementarity problem and the degenerated complementarity problem (4).

The complementarity problem (3) can easily be reduced to the solvability of special systems of two equations. More precisely, the following lemma holds.

Lemma 2. Let $f$ and $g$ be operators in a Hilbert space $X$. Then for each $\lambda \in(0,1)$ the complementarity problem with operators $(1-\lambda) g+\lambda f$ and $g$ is solvable if and only if the following system (with respect to $t, \xi \in X$ )

$$
\left.\begin{array}{r}
f(t)+\xi-\lambda P_{K} \xi=0 \\
g(t)-\lambda P_{K} \xi=0
\end{array}\right\}
$$

is solvable.

Proof. The case $\lambda=0$ is evident and so we consider only the case $0<$ $\lambda \leq 1$. Let $\left(t_{*}, \xi_{*}\right)$ be a solution of system (5), i.e.

$$
\left.\begin{array}{r}
f\left(t_{*}\right)+\xi_{*}-\lambda P_{K} \xi_{*}=0 \\
g\left(t_{*}\right)-\lambda P_{K} \xi_{*}=0
\end{array}\right\}
$$

Then $f\left(t_{*}\right)-g\left(t_{*}\right)+\xi_{*}=0$ or, by Lemma 1 ,

$$
(1-\lambda) g\left(t_{*}\right)+\lambda f\left(t_{*}\right)=\lambda\left(P_{K} \xi_{*}-\xi_{*}\right)=-\left(\lambda \xi_{*}-P_{K}\left(\lambda \xi_{*}\right)\right)
$$

Moreover, $g\left(t_{*}\right)=P_{K}\left(\lambda \xi_{*}\right)$. Thus, we get the equations

$$
\begin{aligned}
(1-\lambda) g\left(t_{*}\right)+\lambda f\left(t_{*}\right) & =\lambda\left(P_{K} \xi_{*}-\xi_{*}\right)=-\left(\zeta_{*}-P_{K} \zeta_{*}\right) \\
g\left(t_{*}\right) & =P_{K} \zeta_{*}
\end{aligned}
$$

where $\zeta_{*}=\lambda \xi_{*}$. But, by Lemma 1 ,

$$
P_{K} \zeta_{*} \in K, \quad-\left(\zeta_{*}-P_{K} \zeta_{*}\right) \in K^{*}, \quad\left(P_{K} \zeta_{*},-\left(\zeta_{*}-P_{K} \zeta_{*}\right)\right)=0
$$

and, consequently, these equations, again by Lemma 1, immediately imply that $t_{*}$ is a solution to the complementarity problem with the same $\lambda$.

All these considerations can be inverted. This means that if $\left(t_{*}, \xi_{*}\right)$ is a solution of system $(5)$, then $t_{*}$ is a solution of the complementarity problem with operators $(1-\lambda) g+\lambda f$ and $g$ 
It is evident that system (5) is equivalent to the system

$$
\left.\begin{array}{r}
f(t)-g(t)+\xi=0 \\
g(t)-\lambda P_{K} \xi=0
\end{array}\right\}
$$

but this system is equivalent to the equation

$$
g(t)-\lambda P_{K}(g(t)-f(t))=0 .
$$

Thus, we have the following

Lemma 3. Let $f$ and $g$ be operators in a Hilbert space $X$. Then, for each $\lambda \in(0,1), t_{*}$ is a solution of the complementarity problem with operators $(1-\lambda) g+\lambda f$ and $g$ if and only if it is a zero of the vector field

$$
\Psi(\lambda)(t)=g(t)-\lambda P_{K}(g(t)-f(t)) .
$$

Unfortunately, in general the vector fields of the family $\Psi(\lambda) \quad(0 \leq \lambda \leq 1)$ do not belong to a class of fields for which the degree is defined. However, it is a possible to pass from the family of vector fields $\Psi(\lambda) \quad(0 \leq \lambda<1)$ to another family $H(\lambda) \quad(0 \leq \lambda<1)$ of class $S_{+}$; remark that this passage is not possible for the field $\Psi(1)$, but the passage $\Psi(\lambda) \rightarrow H(\lambda) \quad(0 \leq \lambda<1)$ is sufficient for our considerations.

Remark that for each $\lambda \in[0,1)$ the operator $\left(I-\lambda P_{K}\right)^{-1}$ exists and is bounded and continuous. This follows from the fact that the operator $P_{K}$ is non-expansive (see Lemma 1). The evident chain of equations

$$
\begin{aligned}
\Psi(\lambda)= & g(t)-\lambda P_{K}(g(t)-f(t)) \\
= & \left(I-\lambda P_{K}\right)(g(t)-f(t))+f(t) \\
= & \left(I-\lambda P_{K}\right)\left(g(t)-f(t)+\left(I-\lambda P_{K}\right)^{-1} f(t)\right) \\
= & \left(I-\lambda P_{K}\right) H(\lambda) \\
& \left(0 \leq \lambda<1, H(\lambda)=g-\left(I-\lambda P_{K}\right)^{-1} f\right)
\end{aligned}
$$

implies the following

Lemma 4. Let $f$ and $g$ be operators in a Hilbert space $X$. Then for each $\lambda \in(0,1), t_{*}$ is a solution of the complementarity problem with operators $(1-\lambda) g+\lambda f$ and $g$ if and only if it is a zero of the vector field

$$
H(\lambda)(t)=g(t)-f(t)+\left(I-\lambda P_{K}\right)^{-1} f(t) .
$$

Recall that a vector field $H$ in a Hilbert space $X$ is demicontinuous if it maps sequences convergent in norm in weakly convergent sequences; it is called of class $S_{+}$if each sequence $\left(x_{n}\right)$ from $X$, which weakly converges to $x_{*}$ and satisfies the condition

$$
\limsup _{n \rightarrow \infty}\left(H x_{n}, x_{n}-x_{*}\right) \leq 0
$$

converges to $x_{*}$ in norm.

The following lemma is almost evident. 
Lemma 5. Let $f$ be completely continuous and $g$ of class $S_{+}$in a Hilbert space $X$. Then for each $\lambda \in[0,1)$ the operator $H(\lambda)$ is of class $S_{+}$.

This lemma shows that we can use the Skrypnik degree in order to study all vector fields $H(\lambda) \quad(0 \leq \lambda<1)$ whose zero by Lemmas 3 and 4 are solutions of the complementarity problems with operators $(1-\lambda) g+\lambda f \quad(0 \leq \lambda<1)$ and $g$.

The Skrypnik theory [10] (see also [8]) states that for each demicontinuous field $\Phi$ of class $S_{+}$defined on a bounded domain $\Omega$ and without zeroes on the boundary $\partial \Omega$ of the domain $\Omega$ there is defined an integer $\gamma(\Phi, \Omega)$ (the degree of the field $\Phi$ on the boundary $\partial \Omega$ of the domain $\Omega$ ), and the function

$$
(\Phi, \Omega) \rightarrow \gamma(\Phi, \Omega)
$$

has the usual properties of Brouwer-Hopf and Leray-Schauder degree: $X)$.

I. $\gamma(I, \Omega)=1$ if $0 \in \Omega$ where $I$ is the identity mapping, i.e. $I t=t \quad(t \in$

II. If $\Omega=\Omega_{1} \cup \Omega_{2}$ and $\Phi$ has no zero on the set $\partial \Omega_{1} \cup \partial \Omega_{2} \cup\left(\Omega_{1} \cap \Omega_{2}\right)$, then $\gamma(\Phi, \Omega)=\gamma\left(\Phi, \Omega_{1}\right)+\gamma\left(\Phi, \Omega_{2}\right)$.

III. If $\Phi_{0}$ and $\Phi_{1}$ are homotopic on $\Omega$, then $\gamma\left(\Phi_{0}, \Omega\right)=\gamma\left(\Phi_{1}, \Omega\right)$ (vector fields $\Phi_{0}$ and $\Phi_{1}$ are homotopic on $\Omega$ if there exists a family $\Phi(\lambda, \cdot) \quad(0 \leq \lambda \leq 1)$ of class $S_{+}$, defined on $\bar{\Omega}$ and demicontinuous with respect to both variables such that $\Phi(0, \cdot)=\Phi_{0}, \Phi(1, \cdot)=\Phi_{1}$ and $\left.\Phi(\lambda, t) \neq 0 \quad(0 \leq \lambda \leq 1, t \in \partial \Omega)\right)$.

In the theory of Skrypnik degree the following analogue of the basic existence principle holds:

If $\Phi$ has no zero on the boundary $\partial \Omega$ of the domain $\Omega$ and the degree $\gamma(\Phi, \Omega)$ of this vector field on the boundary $\partial \Omega$ of $\Omega$ is non-zero, then there exists at least one zero $t_{*}$ of $\Phi$ in $\Omega$.

As was proved above, the vector fields $H(\lambda)(0 \leq \lambda<1)$ defined by (8) are of class $S_{+}$. Consider the family of vector fields $H(\lambda)(0 \leq \lambda<1)$ on a domain $\Omega$. It is evident that the family under our assumptions is demicontinuous with respect to both variables and $H(0, \cdot)=g$. Assume that $\gamma(g, \Omega) \neq 0$.

We have two possibilities:

- First, there exist $\lambda_{*} \in(0,1)$ and $t_{*} \in \partial \Omega$ such that $H\left(\lambda_{*}\right) t_{*}=0$. In this case $t_{*}$ is a solution of the complementarity problem with the operators $\left(1-\lambda_{*}\right) g+\lambda_{*} f$ and $g$.

- Second, for all $\lambda \in(0,1)$ the inequalities $H(\lambda) x \neq 0 \quad(x \in \partial \Omega)$ hold. Then all vector fields $H(\lambda) \quad(0 \leq \lambda<1)$ are homotopic on $\Omega$ and, therefore, they have the same degree $\gamma(H(\lambda), \Omega)$ on the boundary $\partial \Omega$ of the domain $\Omega$. 
But $\gamma(H(0), \Omega) \neq 0$ by assumption. Thus, in the second case we have the equation

$$
\gamma(H(\lambda), \Omega) \neq 0 \quad(0 \leq \lambda<1) .
$$

This implies that each vector field $H(\lambda) \quad(0 \leq \lambda<1)$ has at least one zero $t_{\lambda} \in \Omega$ :

$$
g\left(t_{\lambda}\right)=\lambda P_{K}\left(g\left(t_{\lambda}\right)-f\left(t_{\lambda}\right)\right)
$$

This implies the solvability of all complementarity problems with operators $(1-\lambda) I+\lambda f \quad(0 \leq \lambda<1)$ and $g$ in the domain $\Omega$. Moreover, if $g$ is a bounded operator, then the equation

$$
g\left(t_{\lambda}\right)-P_{K}\left(g\left(t_{\lambda}\right)-f\left(t_{\lambda}\right)\right)=(1-\lambda) P_{K}\left(g\left(t_{\lambda}\right)-f\left(t_{\lambda}\right)\right)
$$

implies

$$
\inf _{t \in \Omega}\left\|g(t)-P_{K}(g(t)-f(t))\right\|=0
$$

This means that the original complementarity problem is "almost" solvable in this case.

Let $\left(\lambda_{n}\right) \rightarrow 1$ and $t_{n}=t_{\lambda_{n}} \quad(n \geq 1)$. Without loss of generality we can assume that the sequence $\left(g\left(t_{n}\right)\right)$ weakly converges to a $w_{*}$ and the sequence $\left(f\left(t_{n}\right)\right)$ converges in norm to a $v_{*}$. Since $\Psi(1)\left(t_{n}\right)=g\left(t_{n}\right)-P_{K}\left(g\left(t_{n}\right)-f\left(t_{n}\right)\right)$, $\left\|\Psi(1)\left(t_{n}\right)\right\| \rightarrow 0$ and

$$
P_{\left(-K^{*}\right)}\left(g\left(t_{n}\right)-f\left(t_{n}\right)\right)=\left(I-P_{K}\right)\left(g\left(t_{n}\right)-f\left(t_{n}\right)\right)=\Psi(1)\left(t_{n}\right)-f\left(t_{n}\right),
$$

the sequence $\left(P_{\left(-K^{*}\right)}\left(g\left(t_{n}\right)-f\left(t_{n}\right)\right)\right)$ converges in norm to $-v_{*}$. On the other hand, the sequence $\left(g\left(t_{n}\right)-f\left(t_{n}\right)\right)$ weakly converges to $w_{*}-v_{*}$. But this (see Lemma 1) implies

$$
P_{\left(-K^{*}\right)}\left(w_{*}-v_{*}\right)=-v_{*} \cdot
$$

Now, if we assume that $f$ and $g$ are weakly continuous operators, we obtain the equality

$$
P_{\left(-K^{*}\right)}\left(g\left(t_{*}\right)-f\left(t_{*}\right)\right)+f\left(t_{*}\right)=0
$$

or $\Psi(1)\left(t_{*}\right)=0$. This implies the solvability of the original complementarity problem with operators $f$ and $g$.

One can weaken the assumptions about weak continuity of $f$ and $g$. Equation (11) can be rewritten in the form

$$
w_{*} \in v_{*}+P_{\left(-K^{*}\right)}^{(-1)}\left(-v_{*}\right)
$$

where $P_{\left(-K^{*}\right)}^{(-1)}(\cdot)$ denotes the pre-image of $(\cdot)$ under the mapping $P_{\left(-K^{*}\right)}$. Thus, by our considerations, we see that the sequence $\left(t_{n}\right)$ weakly converges to $t_{*}$ 
such that $\left(f\left(t_{n}\right)\right)$ converges in norm to $v_{*} \in-K^{*}, g\left(t_{n}\right) \in K \quad(n \geq 1)$ and $\left(g\left(t_{n}\right)\right)$ weakly converges to $w_{*} \in v_{*}+P_{\left(-K^{*}\right)}^{(-1)}\left(-v_{*}\right)$. We call the pair of operators $f$ and $g$ regular if all these conditions imply $f\left(t_{*}\right)=v_{*}$ and $g\left(t_{*}\right)=w_{*}$.

We summarize all statements obtained as a result of these considerations in the form of a theorem.

Theorem 3. Let $f$ be completely continuous, $g$ of class $S_{+}$, and the pair $f, g$ regular. Let $\Omega$ be a bounded domain and $\gamma(g, \Omega) \neq 0$. Then

- either for some $\lambda \in(0,1)$ the complementarity problem with the operators $(1-\lambda) g+\lambda f$ and $g$ has a solution on the boundary $\partial \Omega$ of the domain $\Omega$

- or the complementarity problem with the operators $f$ and $g$ has a solution in the domain $\Omega$.

We can also formulate an analogue of [1: Theorem 2]. Put

$$
\begin{aligned}
& \widetilde{f}(t)=\left\{\lim _{n \rightarrow \infty} f\left(t_{n}\right):\left(t_{n}\right) \in \mathcal{W}(t)\right\} \\
& \widetilde{g}(t)=\left\{\lim _{n \rightarrow \infty} g\left(t_{n}\right):\left(t_{n}\right) \in \mathcal{W}(t)\right\}
\end{aligned}
$$

where $\mathcal{W}(t)$ is a set of sequences $\left(t_{n}\right)$ with elements $t_{n}$ from $X$ which weakly converge to $t$, and such that $\left(f\left(t_{n}\right)\right)$ converges in norm to some $\phi \in K^{*}$, $g\left(t_{n}\right) \in K(n \geq 1),\left(g\left(t_{n}\right)\right)$ weakly converges to $\psi \in K$ and $\left(g\left(t_{n}\right), f\left(t_{n}\right)\right) \leq$ $0 \quad(n \geq 1)$.

Using these notations we can consider the following generalized complementarity problems with multi-valued operators $\widetilde{f}$ and $\widetilde{g}$ :

$$
\psi \in K, \quad \phi \in K^{*}, \quad(\psi, \phi)=0, \quad \phi \in \widetilde{f}, \quad \psi \in \widetilde{g} .
$$

The following result is evident:

Theorem 4. Let $f$ be completely continuous and $g$ of class $S_{+}$. Let $\Omega$ be a bounded domain, whose closure $\bar{\Omega}$ is weakly sequentially closed, and $\gamma(g, \Omega) \neq 0$. Then

- either for some $\lambda \in(0,1)$ the complementarity problem with the operators $(1-\lambda) g+\lambda f$ and $g$ has a solution on the boundary $\partial \Omega$ of the domain $\Omega$

- or the generalized complementarity problem with the operators $\widetilde{f}$ and $\widetilde{g}$ has a solution in the domain $\Omega$. 
3. In the finite-dimensional case Theorem 3 is similar to [6: Theorem 7]. However, the authors of [6] used homotopies which are different from family (7). In our notation they considered the family

$$
\Psi_{0}(\lambda)(t)=g(t)-\lambda P_{K}(g(t)-\lambda f(t)) \quad(0 \leq \lambda \leq 1) .
$$

This family is generated by the system

$$
\left.\begin{array}{rl}
\lambda f(t)+\xi-\lambda P_{K} \xi & =0 \\
g(t)-\lambda P_{K} \xi & =0
\end{array}\right\} \quad(0 \leq \lambda \leq 1)
$$

which is different from system (5); however, for $\lambda=1$ they both coincide. One can see that system (13) is equivalent to the family of complementarity problems

$$
\begin{gathered}
g(t) \in K, \quad \frac{1-\lambda}{\lambda} g(t)+\lambda f(t) \in K^{*}, \quad\left(g(t), \frac{1-\lambda}{\lambda} g(t)+\lambda f(t)\right)=0 \\
(0<\lambda \leq 1) .
\end{gathered}
$$

Of course, one can repeat all our considerations with the family $\Psi_{0}(\lambda) \quad(0 \leq$ $\lambda \leq 1)$ instead of the family $\Psi(\lambda) \quad(0 \leq \lambda \leq 1)$; in this case it is necessary to consider the family of vector fields

$$
H_{0}(\lambda)=g-\lambda f+\left(I-\lambda P_{K}\right)^{-1}(\lambda f) \quad(0 \leq \lambda \leq 1) .
$$

The corresponding analysis is complicated by the fact that the complementarity problem (14) is not defined for $\lambda=0$.

In conclusion, we discuss the passage to exceptional families of elements for both approaches. Let $t_{*}$ be a solution to the complementarity problem (3) for a fixed $\lambda \in(0,1)$ :

$$
g\left(t_{*}\right) \in K, \quad(1-\lambda) g\left(t_{*}\right)+\lambda f\left(t_{*}\right) \in K^{*}, \quad\left(g\left(t_{*}\right),(1-\lambda) g\left(t_{*}\right)+\lambda f\left(t_{*}\right)\right)=0 .
$$

Put

$$
w=(1-\lambda) g\left(t_{*}\right)+\lambda f\left(t_{*}\right) .
$$

Then one can write

$$
f\left(t_{*}\right)=-\frac{1-\lambda}{\lambda} g\left(t_{*}\right)+\omega
$$

where $\omega=\lambda^{-1} w$. The element $\omega$ has the properties

$$
\omega \in K^{*}, \quad(u, \omega)=0 \quad\left(u \in \Pi\left(t_{*}\right)\right)
$$

where

$$
\Pi\left(t_{*}\right)=\left\{u \in K: \ell(u)=0 \quad\left(\ell \in K^{*}, \ell\left(g\left(t_{*}\right)\right)=0\right)\right\} .
$$


This means that $t_{*}$ is an exceptional element for the original complementarity problem (2).

Similarly, let $t_{*}$ be a solution to the complementarity problem (14) for a fixed $\lambda \in(0,1)$ :

$$
g\left(t_{*}\right) \in K, \quad \frac{1-\lambda}{\lambda} g\left(t_{*}\right)+\lambda f\left(t_{*}\right) \in K^{*}, \quad\left(g\left(t_{*}\right), \frac{1-\lambda}{\lambda} g\left(t_{*}\right)+\lambda f\left(t_{*}\right)\right)=0 .
$$

In this case we put

$$
w=\frac{1-\lambda}{\lambda} g\left(t_{*}\right)+\lambda f\left(t_{*}\right)
$$

Then

$$
f\left(t_{*}\right)=-\frac{1-\lambda}{\lambda^{2}} g\left(t_{*}\right)+\omega
$$

where $\omega=\lambda^{-1} w$. The element $\omega$ satisfies property (15) and this means that $t_{*}$ is an exceptional element for the original complementarity problem (2).

One can see that the difference in (15) and (17) is only in notation of the positive parameter $\mu$ which is defined by $\mu=\frac{1-\lambda}{\lambda}$ and $\mu=\frac{1-\lambda}{\lambda^{2}}$, correspondingly. Thus, in the finite-dimensional case, Theorem 3 and [6: Theorem 7] both state the existence of exceptional families of elements in the case when the original complementarity system is not solvable. However, in the framework of complementarity theory, these theorems state the solvability of auxiliary complementarity problems with different operators.

\section{References}

[1] Carbone, A. and P. P. Zabreı̌ko: Some remarks on complementarity problems in a Hilbert space. Z. Anal. Anw. 21 (2002), 1005 - 1014.

[2] Hyers, D. H., Isac, G. and T. M. Rassias: Topics in Nonlinear Analysis 86 Applications. Singapore: World Sci. 1997.

[3] Isac, G.: Fixed point theory, coincidence equations on convex cones and complementarity problem. Contemp. Math. 72 (1988), 139 - 155.

[4] Isac, G.: Complementarity Problems. Lect. Notes Math. 1528 (1992), 139 155.

[5] Isac, G.: Topological Methods in Complementarity Theory. Dordrecht: Kluwer Acad. Publ. 2000.

[6] Isac, G., Bulavski, V. and V. Kalashnikov: Exceptional families, topological degree and complementarity problems. J. Global Optim. 10 (1997), 207 - 225.

[7] Isac, G. and A. B. Németh: Projection methods, isotone projection cones and the complementarity problem. J. Math. Anal. Appl. 153 (1990), 258 - 275.

[8] Krasnosel'skiı̌, M. A. and P. P. Zabreı̌ko: Geometrical Methods of Nonlinear Analysis. Berlin: Springer-Verlag 1984. 
[9] Moreau, J.: Décomposition orthogonale d'un espace hilbertien selon deux cônes mutuellement polaires. C.R. Acad. Sci. Paris 225 (1962), 238 - 240.

[10] Skrypnik, I. V.: Methods for Analysis of Nonlinear Elliptic Boundary Value Problems (Transl. Math. Mon.: Vol. 139). Providence (R.I., USA): Amer. Math. Soc. 1994.

Received 15.02.2002 